\title{
THE FIFTY-THIRD ANNUAL MEETING OF THE AMERICAN ASSOCIATION FOR THE ADVANCEMENT OF SCIENCE.
}

The fifty-third annual meeting of the American Association for the Advancement of Science was held at St. Louis during the convocation week, December 28, 1903, to January 2, 1904.

Honorable Carroll D. Wright was president. The address of the retiring president, Dr. Ira Remsen, entitled "Scientific investigation and progress," was given at the Odeon on the evening of the opening day. Though the enrollment was small, reaching a total of only 466 , the meeting was in every other respect an eminently successful one. The cause of the small attendance is not to be sought in any general diminution of interest in the work of the Association; but rather in the fact that many have preferred to visit St. Louis a few months hence, when the Congress of Arts and Sciences to be held in connection with the Louisiana Purchase Exposition shall be in session.

Excellent accommodations were provided for all the sections and for the twenty-one affiliated societies in the Central High School Building. Everything that could contribute to the comfort and convenience of visiting scientists had been anticipated by a most efficient local committee. Of the affiliated societies, the Astronomical and Astrophysical Society of America and the Chicago Section of the American Mathematical SocIETY held joint sessions with Section A.

As compared with previous years, but few mathematicians and astronomers were present and there was a correspondingly small number of papers for the programme of Section A. The officers of the section were : vice-president, Otto H. Tittmann ; secretary, L. G. Weld ; councilor, Ormond Stone; sectional committee, G. B. Halsted, C. S. Howe, W. W. Beman, J. A. Brashear, J. R. Eastman, Ormond Stone and E. B. Frost, together with the president and the secretary; member of the general committee, Philip Fox. The following mathematicians and astronomers were elected by the Council to fellowship in the Association: Luigi d'Auria, G. E. Fisher, B. F. Groat, Edward Kasner, G. W. Littlehales, and F. L. O. Wadsworth. 
The vice-presidential address for Section A, by Professor G. B. Halsted, was upon the subject "The message of non-euclidean geometry." It will be published in Science.

The following list of the papers presented does not include those of the Astronomical and Astrophysical Society and of the Chicago Section of the American Mathematical Society which were read at the joint sessions of these societies and Section A.

(1) Professor G. B. Halsted : "A new treatment of volume."

(2) Mr. E. L. HANCOCK : "Lines on the pseudosphere and the syntractrix of revolution."

(3) Professor G. W. Hough : "On the rotation period of the planet Saturn."

(4) Dr. Edward Kasner: "An extension of the group concept."

(5) Mr. E. L. Larkin : "Facilities for astronomical photography. in southern California."

(6) Mr. L. S. McCoy : "Coincident variations."

(7) Professor G. A. Miller : "On the generalization and extension of Sylow's theorem."

(8) Professor C. D. Perrine : "The supporting and counter-weighting of the principal axes of large telescopes."

(9) Mr. J. J. QuINN : "A linkage for describing the conic sections by continuous motion."

(10) Mr. T. R. RunNing: "Circles represented by $\mu^{3} P$ $+L \mu^{2} Q+M \mu R+N S=0 . "$

(11) Professor David Todd : "A new type of transit-room shutter."

In the absence of the authors the papers by Mr. Hancock, Dr. Miller, Professor Perrine, Mr. Quinn, Mr. Running and Professor Todd were presented in outline by the secretary. The papers by Dr. Kasner and Mr. McCoy were read by title. Dr. Miller's paper will soon be printed in full in the American Mathematical Monthly. Abstracts, as far as available, of such of the above papers as deal with mathematical subjects, are given below.

1. Professor Halsted quotes from Poincaré's review of Hilbert's Grundlagen der Geometrie as follows :

"The fourth book treats of the measurements of plane areas. If this measurement can be easily established without the aid 
of the principle of Archimedes, it is because two equivalent polygons can either be decomposed into triangles in such a way that the component triangles of the one and those of the other are equal each to each, or else can be regarded as the difference of polygons capable of this mode of decomposition. But we must observe that an analogous condition does not seem to exist in the case of two equivalent polyhedrons; so that it becomes a question whether or not we can determine the volume of the pyramid, for example, without an appeal more or less disguised to the infinitesimal calculus. It is, therefore, not certain that we can dispense with the axiom of Archimedes in the measurement of volumes. Moreover, Professor Hilbert has not attempted it."

Professor Halsted, in his paper, attacks the problem in the following manner :

The product of an altitude of a tetrahedron by the area of its base is the same whichever of the four faces may be chosen as base. This product is, therefore, a "natural invariant" of the tetrahedron and may be designated as its volume, except that in order to adjust the conception to our ordinary numerical scale the factor one third is arbitrarily introduced. After defining a transversal partition of a tetrahedron as one made by a plane through an edge and a point of the opposite edge, it was shown that, however this solid be cut by a plane, the partition can be obtained as a result of successive transversal partitions, using not more than two other planes.

The above being explained, it was shown that the volume of any tetrahedron is equal to the sum of the volumes of all tetrahedrons which result from any set of transversal partitions. This need not be assumed as self-evident, but may be demonstrated as a necessary consequence of the so-called "betweenness" assumption with reference to three collinear points. Similar principles were deduced for polyhedrons in general, and by their use a general theory of volume was built up without reference to the ordinary notions of ratio and commensurability. The same method of treatment may be applied to figures in hyperspace of any order.

2. In Mr. Hancock's paper, the lines of the pseudosphere are reviewed and those of the syntractrix of revolution studied. The latter surface $S_{1}$ is defined as the surface generated by the revolution of the curve $C_{1}$ about its asymptote ; $C_{1}$ being de- 
termined by laying off a constant distance $d$ on the tangents of the tractrix.

The geodesic, asymptotic and loxodromic lines on $S_{1}$ are worked out and studied by classifying the surfaces according as

$$
d \geqq 2 c,
$$

$c$ being the constant of the tractrix. When $d \geqq 2 c$ it happens that the geodesic lines on $S_{1}$ are all real; while for $d<2 c$ they are real or imaginary according as

$$
\kappa^{2} \lessgtr\left|\frac{c^{2} d^{2}}{d^{2}-4 c d}\right|
$$

$\kappa$ being a constant of integration.

The loxodromic lines of the syntractrix of revolution are represented in the plane by the same system of straight lines as represent the loxodromic lines of the pseudosphere.

3. Professor Hough gives the following account of his recent determination of the rotation period of the planet Saturn :

In 1877 Professor Asaph Hall, then at the U. S. Naval Observatory, observed a spot near to Saturn's equator and by its means determined the period of the planet's rotation. From that time on, until the recent opposition, no well-defined spot had been visible. On June 23, 1903, however, Professor E. E. Barnard, of the Yerkes Observatory, noted a large and distinct spot in kronocentric latitude $36^{\circ} .5$. This was observed micrometrically on June 27 and July 13.

Acting upon the request of the author, micrometric observations of spots on Saturn were make by Professor S. W. Burnham with the 40-inch Yerkes equatorial. Measurements were secured on July 29 and August 15. From these data the " mean" rotation period deduced was $10^{\mathrm{h}} 38^{\mathrm{m}} 27^{\mathrm{s}}$; but the observations showed the period to be variable. The value $10^{\mathrm{h}}$ $38^{\mathrm{m}} 18^{\mathrm{s}}+n \times 0^{\mathrm{s}} .1856$ was found to satisfy all the observations with a mean error of $\pm 0^{\mathrm{m}} .8$. In the formula $n$ is the number of rotations of the planet counting from the epoch of the discussion, June 23, 1903.

4. The essential property of a group is that its elements form a closed system, that is, the combination of any two of the elements is equivalent to an element of the system. There arise, 
however, in different fields of mathematics, systems of elements such that the combination of any three (though not of any two) is a member of the system. The following are examples of such triadic systems: the odd permutations in any number of letters, the $\infty^{2}$ central symmetries of the plane or the $\infty^{3}$ of space, the totality of dual or reciprocal transformations, the correlations contained in any projective group, the totality of conformal transformations of the plane which reverse angles. Dr. Kasner investigates such systems from the abstract point of view, and, more generally, systems for which the combination of any $k$ (though not of fewer) is contained in the system.

It is proposed to term such a system a closed system of connectivity $k$, or more simply, in analogy with the nomenclature of the logic of relations, a $k$-adic system. The ordinary groups are identical, of course, with the dyadic systems. $\dot{A}$ trivial example of a $k$-adic system is that containing as its sole element a primitive $(k-1)$ th root of unity, combination being interpreted as ordinary multiplication. The law of combination for the general system is best exhibited by means of its $k$ dimensional multiplication table.

The paper discusses in particular the relation of systems to groups. No system of connectivity greater than two can contain an identical element. If a system contains the inverse of each of its elements, as is the case in the examples mentioned at the outset, its connectivity must be either two or three; so that, in addition to the ordinary groups, the only systems having this property are triadic. Furthermore it is possible to regard such a triadic system as the half of an ordinary group.

7. The scope and nature of Professor Miller's extension of Sylow's theorem can best be explained by quoting his own abstract of the paper, which reads :

Let $p^{\alpha}$ be the highest power of $p$ which divides the order of a group $(G)$, and suppose that a subgroup $\left(P_{\alpha}\right)$ of order $p^{\alpha}$ contains only one subgroup $\left(P_{\beta}\right)$ of order $p^{\beta}$ and of a particular type. It is proved that the number of subgroups of $G$ which are of the same type as $P_{\beta}$ is of the form $1+k p$ and that all of these subgroups form a single conjugate set. Hence the order of $G$ is of the form $p^{\beta} h_{1}(1+k p)$ where $p^{\beta} h_{1}$ is the order or the largest subgroup of $G$ which transforms $P_{\beta}$ into itself. By letting $\beta=\alpha$ we have Sylow's theorem. When $\beta=\alpha$ the 
factor $h_{1}$ is not divisible by $p$ while it is divisible by $p$ for all other values of $\beta$. Some simplifications of the proof of Frobenius's extension of Sylow's theorem are also considered.

9. The linkage described by Mr. Quinn is the material embodiment of the facts set forth in the following theorems :

If one vertex of a movable pivoted rhombus be fixed in position, while the opposite vertex is constrained to move in the arc of a circle, the locus of the intersection of a diagonal (produced) through the other two vertices, with the radius (produced) of the circle in which the vertex moves is a conic.

If the fixed vertex is in the diameter of the circle, and the directing radius finite, the locus is an ellipse. If the directing radius is infinite and the fixed vertex in the diameter, the locus is a parabola. If the directing radius is finite, and the fixed vertex is in the diameter produced, the locus is a hyperbola.

Modifications of the essential features of this linkage give rise to many interesting corollaries involving the geometric construction of the conies, their tangents and normals.

10. In the equation discussed by Mr. Running $\mu$ represents a variable parameter; $L, M$ and $N$ are constants ; $P$, $Q, R$ and $S$ represent circles. The equation itself represents circles for all values of the parameter. Three circles of the system pass through each point of the plane. The locus of the centers of the system is a cubic having eight arbitrary constants.

There will be a circle orthogonal to the system if any one of the circles $P, Q, R, S$ can be derived linearly from the other three. There are six point circles in the system, all lying upon the locus of the centers. Four circles of the system are tangent to any one. Eight pairs of tangent circles have a common linear relation connecting their parameters.

The envelope of the system is

$$
\begin{aligned}
18 L M N P Q R S-27 N^{2} P^{2} S^{2} & +L^{2} M^{2} Q^{2} R^{2} \\
& -4\left(L^{3} N Q^{3} S+M^{3} P R^{3}\right)=0 ;
\end{aligned}
$$

which may be written $B^{2}=4 A C$, where $A=L^{2} Q^{2}-3 P M R$, $B=L M Q R-9 P N S, C=M^{2} R^{2}-L Q N S$. It is shown that this is the envelope of $\mu^{2} A+\mu B+C=0, A, B, C$ being bi- 
circular quartics which are themselves envelopes of systems derived from the original circles.

The envelope of the radical axes of a particular circle and other circles of the system is a conic. This conic may be said to correspond to the particular circle, and there is such a conic corresponding to every circle of the system. The system of circles represented by

$$
\mu^{3} P+L \mu^{2} Q+M \mu R+N S=0
$$

is called the primary system, and the system of conics corresponding to it in the manner above explained, the secondary system. It is shown that the equation of a conic of the secondary system is of the fourth degree with respect to the parameter and that, therefore, four conics of the secondary system pass through any particular point in the plane.

The equation of the radical axes of two circles, $\mu$ and $\mu^{\prime}$, of the system is

$$
y=\frac{F}{G} x+\frac{H}{G},
$$

$F$ and $H$ being of the fourth degree in $\mu$ and $\mu^{\prime}$ and $G$ of the third degree. It thus appears that there are sixteen sets of values of $\mu$ and $\mu^{\prime}$ for which this equation represents the same radical axis ; that is, there are sixteen pairs of circles having the same radical axis. Moreover, to these thirty-two circles there correspond thirty-two conics of the secondary system, all of which are tangent to the same radical axis.

The paper includes, by way of introduction, a brief discussion of the equation,

$$
\mu^{2} P+L \mu Q+M R=0 .
$$

The next meeting of the Association will be held in Philadelphia during the week beginning December 27, 1904, under the presidency of Professor W. G. Farlow, of Cambridge, Mass. Professor Alexander Ziwet, University of Michigan, will be vice-president of Section A and Professor L. G. Weld, University of Iowa, will continue to act as secretary. New Orleans was recommended as the place of meeting two years hence.

The State University of Iowa, Laenas Gifford Weld. IoWA CIty, Iowa. 